\title{
Experimental identification of modal parameters for the model of a building subjected to short-term kinematic excitation
}

https://doi.org/10.2478/sgem-2018-0030

received November 8, 2018; accepted November 14, 2018.

\begin{abstract}
In this work, the input-output method of dynamic parameters' identification is experimentally tested. A method based on the transformation of a dynamic problem into a static problem by means of integration of the input and output signal was presented. The problem discussed in this article is the identification of the coefficients of stiffness matrices and eigenfrequencies of a discrete dynamic system subjected to kinematic input. The experimental analysis was carried out on a threestorey slab-and-column structure, which constitutes a physical model of a building. The vibrations of the model were excited kinematically by an earthquake simulator. The device has a computer-controlled, movable table top, which can move independently in three directions, that is, horizontally, vertically, and rotationally around the vertical axis.
\end{abstract}

The aim of the experimental studies presented in this work was to determine the dynamic parameters of the model (stiffness, natural frequencies) using the inputoutput method in the time domain. Moreover, the results obtained with this method were compared with the results of experimental modal analysis (EMA) in order to verify their correctness. It was assumed that the movement of the base is horizontal and occurs in one direction. Two shortterm, irregular kinematic excitations of the construction were considered, and the selected results and conclusions from experimental analyses were presented in this work.

Keywords: experimental studies; input-output method; identification of modal parameters; kinematic excitation.

\footnotetext{
*Corresponding author: Krzysztof Majcher, Faculty of Civil Engineering, Wroctaw University of Science and Technology, Wybrzeże Wyspiańskiego 27, 50-370 Wrocław, Poland, E-mail: krzysztof.majcher@pwr.edu.pl
}

\section{Introduction}

There are two basic methods in the structural dynamics: computational and experimental. Both these methods are equally important and used for both newly designed construction structures and the existing ones. The purpose of both methods is the same - they usually allow for determining the basic dynamic features of the structure (e.g., natural frequencies and their corresponding vibration modes, criticaldamping ratios) or for determining the level of construction effort (e.g., by determining the actual dynamic ratio). Computer programs based on the finite element method (FEM) are usually used in relation to a newly designed object, which necessitates dynamic analysis (e.g., a tall building). A computational model is then defined, which subjected to designed dynamic loads, and is analyzed in terms of dynamic response to a given load. When such analyses are insufficient, the engineers decide to carry out additional experimental studies, usually on a model scale. This is the case, for example, when designing a tall building exposed to gusts of wind or earthquakes. Then, the complete dynamic response of the designed object is obtained by using the experimental method in addition to the computational method.

In relation to an existing object, one generally strives to use both methods, so the computational model is formulated as well and subjected to further analysis. However, in the situation when an actual object is available, the value of the measurement results, and thus of the experimental method, is greater. Then, depending on the needs, methods such as experimental modal analysis (EMA), operational modal analysis, or experimental time analysis find application.

The theoretical foundations of the EMA are given, among others, in the work [5], in which the authors discussed, among others, issues such as obtaining modal characteristics, types of measurements, estimation of errors, and algorithms for dealing with a system with a single and multiple degrees of freedom. In the monograph 
[15] discussing experimental dynamic studies of structures, in addition to the EMA, the operational modal analysis is also comprehensively described. Apart from detailed theoretical discussion of experimental methods, the authors provided many examples of the applications of these methods, along with the results of measurements. It is notable that the examples provided are representative of each method and refer to both real objects and models of construction structures.

Owing to their popularity, experimental methods for the dynamics of building structures have been, and continue to be, the subject of many scientific publications. In 1987, one of the most important works by Ljung [8] was published, discussing the theoretical foundations of system identification. In more than 500 pages of this monograph, the author discussed extensively numerous issues, beginning with linear and nonlinear systems through methods of estimating system parameters to end with model validation. Another work of considerable significance in the field of the identification of system parameters is the article [6] by Imai et al., in which the authors analyzed the methods of identifying dynamic systems relating to the linear and nonlinear behavior of constructions loaded with natural excitations such as earthquakes, gusts of wind, or loading by water waves. Particular attention was paid to the methods that could be used in field studies.

Another remarkable work is [9], in which the authors presented one of the algorithms of procedure corresponding to the input-output method. With the recordings of a real earthquake and the dynamic response of the analyzed systems (computational model and a real object), the ERA (Eigensystem Realization Algorithm) was used to determine the basic modal parameters of objects, such as natural frequency and percentage of critical damping. On the basis of the same ERA algorithm in the work [11], the results of numerical analyses and experimental studies carried out on a laboratory model of a shear building were published.

Extensive explanations referring to the identification of system parameters, the processing of recorded signals, and their application to the detection of damages in construction structures were published in the work [1]. The authors cited and explained many theoretical formulations focusing on the theory of system identification, the application of continuous wavelet transform to achieve the impulse response function, and the problems related to different variants in the input-output method.

In the work [12], Pridham and Wilson proposed an algorithm for the identification of modal parameters of constructions, based only on the output signal.
The effectiveness of the algorithm was verified on a computational model, whose vibrations were excited kinematically. The object's natural frequencies, damping ratios, and vibration modes were determined, having only a record of the dynamic response the object available.

Interesting results of experimental research along with the application of the classical and operational modal analysis methods for the identification of dynamic parameters of large industrial facilities were published in $[3,4]$. In turn, Cunha and Caetano in the work [2] presented the evolution of the EMA from the input-output method to the output-only type. The work presents the results of research collected over many years of the authors' experience, related to civil engineering objects such as dam, building, or bridge.

The inspiration for this publication, however, was the work by Jarczewska et al. [7], in which the authors proposed a method for identifying the stiffness of the system or the natural frequencies, based on the inputoutput method in the time domain. The proposed method consists in transforming a dynamic problem into a static one by integrating input and output signals. One of the main assumptions of the method is that the input signal is short term and irregular. The method allows for identifying system parameters in the case of both force and kinematic excitation.

The main aim of this publication is the experimental verification of the theoretical considerations contained in the work [7], regarding the identification of the modal parameters of a system by converting a dynamic problem into a static one. Moreover, on the basis of the conducted studies, an attempt was made to answer the question: to what extent is the method presented in the work [7] effective in practical applications?

\section{Theoretical foundations}

The theoretical considerations presented in this section refer ultimately to the object of experimental studies, which was a model of a three-storey building with a slaband-column structure (see Fig. 1a). The vibrations of the object were excited kinematically, with the movement of the structure's base interpreted as an input signal in the system, being short-term and irregular. It was assumed that the theoretical model, resulting from the physical model, is a discrete system with three degrees of freedom (see Fig. 1b). This assumption is justified by the fact that the structure of the physical model is very similar to a shear building type, in relation to which only transverse (horizontal) vibrations are considered. 
In a general case, the vibrations of the discrete system are described by equations being well known from the source literature $[5,7,9]$

$$
\mathbf{B} \ddot{\mathbf{q}}(t)+\mathbf{C} \dot{\mathbf{q}}(t)+\mathbf{K} \mathbf{q}(t)=\mathbf{F}(t)
$$

where $\mathbf{B}, \mathbf{C}$, and $\mathbf{K}$ are the matrices of inertia, damping, and stiffness of the system, respectively. The input to the system is the vector of excitation forces $\mathbf{F}(t)$, while the system vibrations are described by the set of generalized Lagrange coordinates contained in the vector $\mathbf{q}(t)$.

When the kinematic excitation is considered instead of force input, the total mass movement of the system is composed of the kinematic component $\mathbf{z}(t)$ and the relative component determined by the vector of generalized coordinates. Then the equation of motion describing the vibrations of the discrete system takes the form of

$$
\mathbf{B} \ddot{\mathbf{q}}(t)+\mathbf{C} \dot{\mathbf{q}}(t)+\mathbf{K} \mathbf{q}(t)=-\mathbf{B} \ddot{\mathbf{z}}(t)
$$

According to the theory proposed in the work [7], if the right side of equation (2) is an irregular short-term or even impulsive input, the duration of which, being marked as $T$, is finite and it is contained within the range $T \in[0 ; t]$, then by integrating both the sides of equation (2) in a finite period of the input time and with zero initial conditions (4), the dynamic process can be transformed into a static one, according to the following formulas:

$$
\begin{gathered}
\int_{0}^{t} \mathbf{B} \ddot{\mathbf{q}}(\tau) \mathrm{d} \tau+\int_{0}^{t} \mathbf{C} \dot{\mathbf{q}}(\tau) \mathrm{d} \tau+\int_{0}^{t} \mathbf{K} \mathbf{q}(\tau) \mathrm{d} \tau=-\int_{0}^{t} \mathbf{B} \ddot{\mathbf{z}}(\tau) \mathrm{d} \tau \\
\mathbf{q}(0)=\mathbf{0}, \quad \dot{\mathbf{q}}(0)=\mathbf{0} \\
\int_{0}^{t} \mathbf{B} \ddot{\mathbf{q}}(\tau) \mathrm{d} \tau=\mathbf{B} \ddot{\mathbf{Y}}(t) \\
\int_{0}^{t} \mathbf{C} \dot{\mathbf{q}}(\tau) \mathrm{d} \tau=\mathbf{C} \dot{\mathbf{Y}}(t) \\
\int_{0}^{t} \mathbf{K} \mathbf{q}(\tau) \mathrm{d} \tau=\mathbf{K} \mathbf{Y}(t) \\
-\int_{0}^{t} \mathbf{B} \ddot{\mathbf{z}}(\tau) \mathrm{d} \tau=-\mathbf{B} \mathbf{Z}(t)
\end{gathered}
$$

When calculating the limit of the expressions $(5,6,7,8)$ for the time value proceeding to infinity, which in practice should be understood as a sufficiently long measurement time, the following values are obtained:

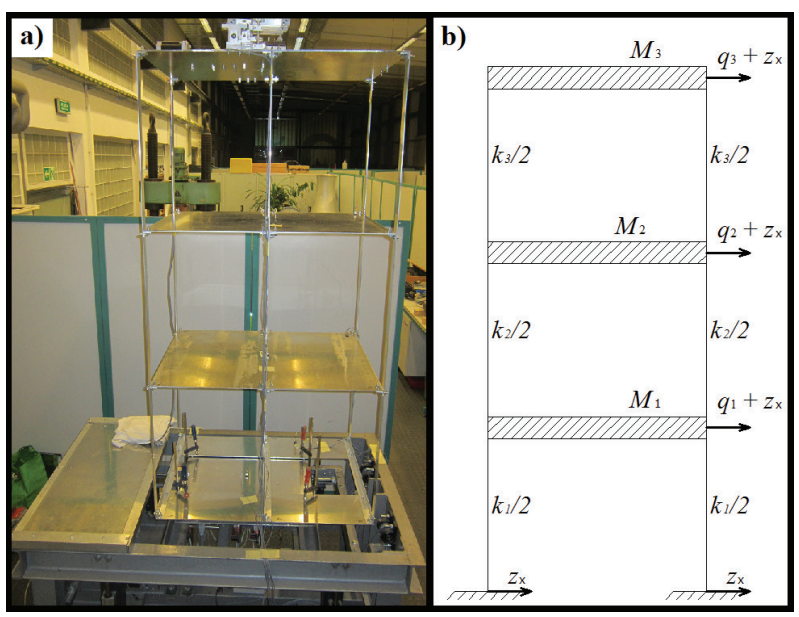

Figure 1: The slab-and-column model: (a) physical; (b) theoretical.

$$
\begin{aligned}
& \lim _{t \rightarrow \infty} \ddot{\mathbf{Y}}(t)=\mathbf{0} \\
& \lim _{t \rightarrow \infty} \dot{\mathbf{Y}}(t)=\mathbf{0} \\
& \lim _{t \rightarrow \infty} \mathbf{Z}(t)=\mathbf{Z}_{c} \\
& \lim _{t \rightarrow \infty} \mathbf{Y}(t)=\mathbf{Y}_{c}
\end{aligned}
$$

In formulas (11) and (12), the right sides of the equation are fixed, time-independent vectors. Therefore, the following equation results from the above dependencies:

$$
\mathbf{K} \mathbf{Y}_{c}=-\mathbf{B ~ Z} \mathbf{Z}_{c}
$$

where $\mathbf{Y}_{c}$ is the time-independent constant vector, which represents static part of structure displacements, and $\mathbf{Z}_{c}$ is the time-independent constant vector, which represents the static part of a structure's mainstay motion.

Equation (13) resembles the classical equation of statics, in which, on the left side, there is a product of the stiffness matrices of the system with a displacement vector, while the right side represents the vector of the forces. Generally, in a typical static problem, the displacement vector is unknown, while the stiffness matrix and the force vector are known. Here it is slightly different, because the vector displacement $\mathbf{Y}_{c}$ is known, while the coefficients of the stiffness matrix are unknown. Equation (13) is a heterogeneous set of algebraic equations that makes it possible to determine these unknown coefficients. In order to determine the desired stiffness matrix of a system whose vibrations are excited kinematically, the inertia matrix $\mathbf{B}$, as well as the function describing the movement of the mainstay structure (vector $\ddot{\mathbf{z}}(t)$ ) and the dynamic 
a)

b)

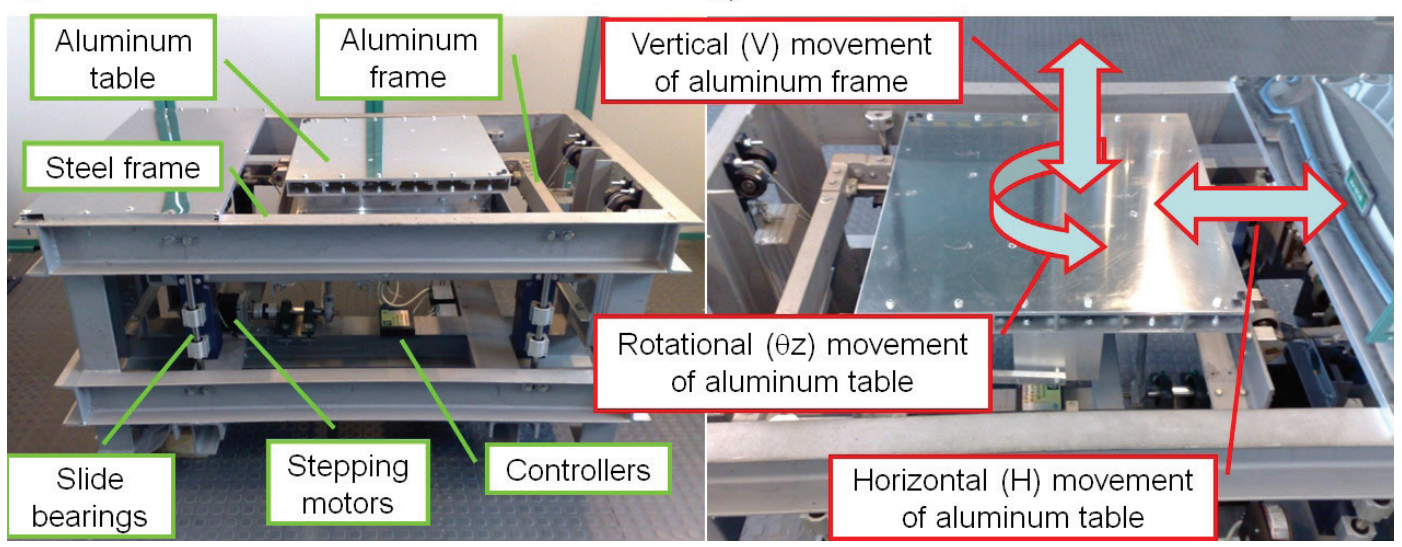

Figure 2: The shake table: (a) main elements and (b) possible directions of kinematic excitation.

response of the system to a pre-set input (vector $\mathbf{q}(t)$ ), must be known.

When the stiffness matrix is identified based on formula (13), the system's natural frequencies can be determined in a standard way by solving the eigenproblem

$$
\operatorname{det}\left(\mathbf{B}^{-1} \mathbf{K}-\{\lambda\} \mathbf{I}\right)=0
$$

where the matrix $\{\lambda\}$ represents a diagonal matrix of the eigenvalues, based on which the natural frequencies are obtained, because $i$ - this eigenvalue is linked to the natural frequency through the following relation

$$
f_{i}=\frac{1}{2 \pi} \sqrt{\lambda_{i}}
$$

\section{Description of the test stand}

Experimental studies consisting in the identification of modal parameters of a system subjected to the kinematic excitations were carried out on a test stand, composed of

- a shake table (see Fig. 2.),

- a model of a three-storey building with a slab-andcolumn structure (see Fig. 1a),

- instruments for measurement-PULSE system by Brüel \& Kjær (see Fig. 3.).

The shake table is a kind of earthquake simulator that allows for mapping seismograms (records of real earthquakes) or any other kinematic excitations. The device used as part of this work has a built-in movable table top on which the models intended for testing are placed (see Fig. 1a). Depending on the needs, the movement of the table top can occur simultaneously in a maximum of three independent directions, that is, horizontal, vertical, and rotational around the vertical axis (see Fig. 2b).

The shake table is characterized by the following technical parameters, important for the mapping of earthquakes:

- the maximum amplitude of vertical and horizontal vibrations is $10 \mathrm{~mm}$,

- the maximum amplitude of rotational vibrations with respect to the vertical axis is 20 degrees,

- the range of frequencies possible to obtain in the generated signal in the horizontal direction is $0.0004-5 \mathrm{~Hz}$, the vertical direction is $0.0004-3 \mathrm{~Hz}$, and the rotational direction is $0.0004-5 \mathrm{~Hz}$,

- all three components of the motion are completely independent of another one (3 degrees of freedom of the table top movement were obtained),

- $\quad$ it is possible to map any course of an earthquake in the range of the given limit values of the amplitude.

The object of experimental research was a model of a three-storey building with a slab-and-column structure, which was built of aluminum sections and plates with the following geometrical and material parameters:

- $\quad$ floor slabs made of aluminum with a dimension of 0.7 $\mathrm{m} \times 0.9 \mathrm{~m} \times 0.005 \mathrm{~m}$ and the following characteristics: $\mathrm{E}=70 \mathrm{GPa}, \mathrm{n}=0.33, \mathrm{r}=2700 \mathrm{~kg} / \mathrm{m}^{3}$,

- dimensions of columns: height $=1.8 \mathrm{~m}$, transverse cross-section $=0.04 \mathrm{~m} \times 0.003 \mathrm{~m}$;

- columns' material: aluminum with the following characteristics: $\mathrm{E}=70 \mathrm{GPa}, \mathrm{n}=0.33, \mathrm{r}=2700 \mathrm{~kg} / \mathrm{m}^{3}$.

The connections of the columns with the ceiling slabs were made with screws in a way that would ensure rigid 
connections. The surface of the floor slab has the shape of a rectangle to which three columns were attached along the long sides-in the corners and in the middle of the side edge. In total, the model of a building consists of six columns and three floor slabs (see Fig. 1a). The masses of the floor slabs, treated as rigid ones, are not the same, and referring to the theoretical model (see Fig. 1b), they are $M_{1}=10.7 \mathrm{~kg}, M_{2}=10.1 \mathrm{~kg}$, and $M_{3}=20.3 \mathrm{~kg}$. Such diversification is influenced by the fact that the building model has a semi-active, tuned mass damper installed on the top floor, which for the purposes of the present experimental research was not dismantled but only turned off and treated as an additional ballast. In the work [10], the results of tests carried out to investigate vibration reduction using a semi-active, tuned mass damper, with the use of the same building model, were published. They include, among others, the results of EMA by means of which the basic natural frequencies of the system were identified. Then the natural frequencies were $f_{1}=0.6 \mathrm{~Hz}$, $f_{2}=2.38 \mathrm{~Hz}, f_{3}=3.93 \mathrm{~Hz}$. However, these tests were carried out in 2013-2014, and since then, the mass of the vibration eliminator has been slightly reduced, which affects the natural frequencies of the entire model-eliminator system. Currently, in the course of EMA, it has been established that the building model has the following natural frequencies: $f_{1}=0.652 \mathrm{~Hz}, f_{2}=2.34 \mathrm{~Hz}, f_{3}=3.92 \mathrm{~Hz}$.

The measurement instruments used for the experimental research were produced by Brüel \& Kjær company. It is a specialized measurement system called PULSE, which is divided into 2 independent sets, and a single module contains

- 17 input channels in the 0 - to $25.6-\mathrm{kHz}$ frequency range,

- 12 channels for slow-changing signals,

- the system and its acquisition modules are made in the Dyn-X technique, that is, they include two 24-bit measurement cards,

- inputs to reach $160-\mathrm{dB}$ dynamics ( $80 \mathrm{~dB}$ per card) and ideal linearity and phase compatibility.

In the system, it is possible to use the transducer electronic data sheet (TEDS) technique, that is, supporting transducers containing automatically read information about themselves (manufacturer, type, serial number) and about the last calibration and place of installation.

The PULSE system includes extensive diagnostics of the state of the input channels that allows for unattended acquisition of signals and does not require paying attention to the adjustment of measuring ranges. The software ensures registering measured signals in parallel with the measurement or only in the form of a recording for

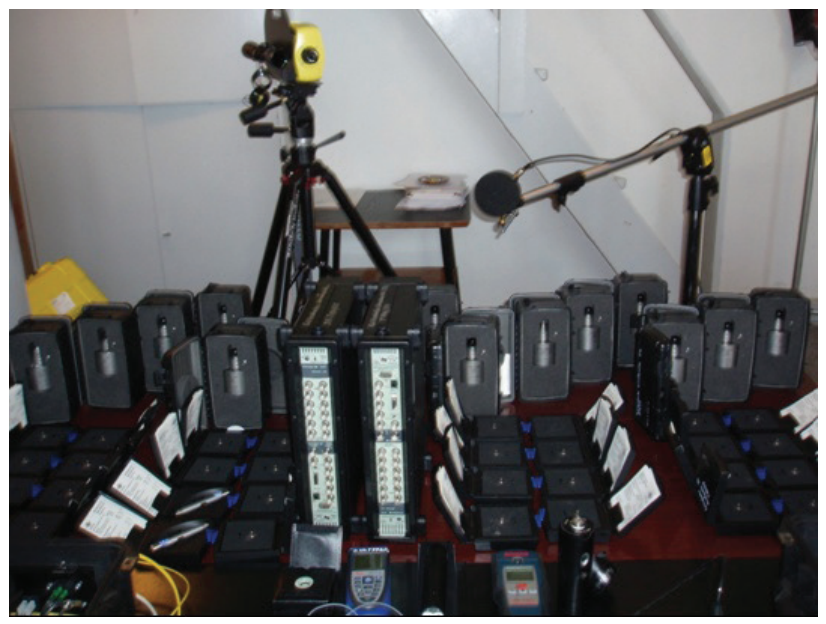

Figure 3: Measurement instruments-PULSE system by Brüel \& Kjær.

further processing; in addition, it ensures registering all kinds of analyses in the scope of vibration measurement and monitoring, modal analysis, noise measurement and monitoring, the sound power of the device, machine diagnostics, and the impact of vibrations on humans.

\section{Experimental research- description and results}

Experimental research consisting in the identification of modal parameters of a three-story building model was carried out on the test stand described in the previous point. The model subjected to testing was placed on the movable table top of the earthquake simulator (see Fig.1a); following this, accelerometers measuring accelerations in the horizontal direction (adequately to the adopted theoretical model) were mounted on each storey of the object (see Fig.1b). The movement of the table top was also recorded using the accelerometer. Table 1 presents the data on kinematic excitations used for the purposes of the experiment. Short-term kinematic inputs were used in the research based on two shapes: half of the sine wave (this input is called "sine"-see Fig. 4a) and a trapezium (the input is called "trapezium"--see Fig. 4b).

According to the theory described in point 2, in order to be able to identify the natural frequencies of the model subjected to kinematic excitations, the masses of the system must be known. Similar to the case of the classical EMA where the value of the force constituting the impulse which induces free vibrations of the system must be known, in the case of a kinematic excitations, the role of the external input force is played by the inertia 
Table 1: Data on kinematic excitations.

\begin{tabular}{llll}
\hline Measurement No. & Name of excitation & $\begin{array}{l}\text { Amplitude of } \\
\text { excitation }(\mathrm{mm})\end{array}$ & $\begin{array}{l}\text { Time duration of } \\
\text { excitation }(\mathbf{s})\end{array}$ \\
\hline 1 & "sine" & 2 & 0.5 \\
2 & "sine" & 4 & 0.5 \\
3 & "trapezium" & 2 & 1 \\
4 & "trapezium" & 4 & 1 \\
5 & "trapezium" & 2 & 2 \\
6 & "trapezium" & 4 & 2 \\
\hline
\end{tabular}

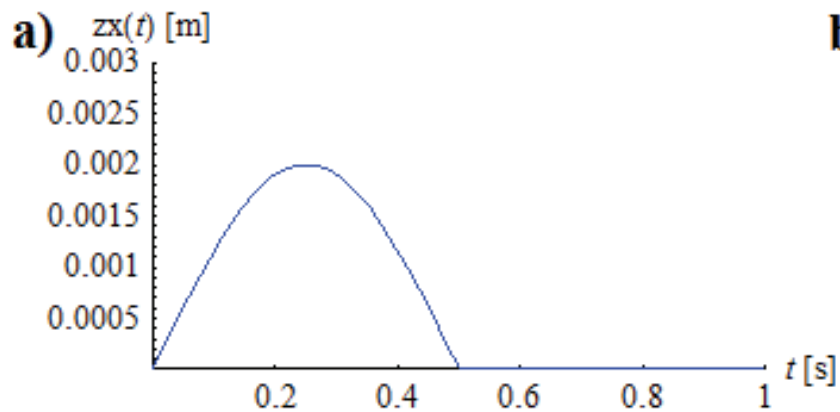

Figure 4: Kinematic excitations: (a) "sine”; (b) "trapezium”.

force, which is internal and its value depends on the mass of the system. Thus, the need to know the masses of the system becomes obvious-they are necessary for a full description of the input affecting the system. In addition, the experimentally verified manner, allowing for the identification of system parameters, is based on the input-output method. Therefore, in addition to the excitation forces, the dynamic response of the system to a given input must be known.

In this work, the results of experimental research were obtained each time using the following procedure:

1. Eliminating any external stimuli that could cause undesirable vibrations of the model-this procedure is related to the theoretical assumption with zero initial conditions (4).

2. Forcing short-term vibrations through the movement of the mainstay structure ("sine" or "trapezium" according to Table 1.) with simultaneous registering of the acceleration of measurement points.

3. Integrating the dynamic model response to obtain the displacement function-according to the theory, the knowledge of the displacement vector $\mathbf{q}(t)$ is required.

4. Calculating integrals according to formulas (7) and (8), and then the limits of expressions according to formulas (11) and (12).

5. Determining stiffness matrix coefficients based on the relation (13).

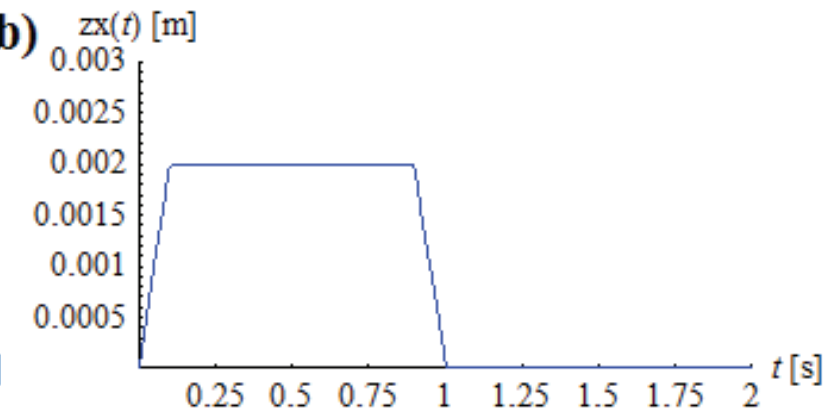

6. Solving the eigensystem (14) and determining the natural frequency of the model (15), based on the stiffness matrix determined experimentally in the previous step.

In point 2 of this article, the theoretical model was adopted as a discrete system with three dynamic degrees of freedom (see Fig. 1b). According to the markings shown in Fig. $1 \mathrm{~b}$ and the geometrical and material data shown in point 3, the inertia matrix of the system has the form

$$
\mathbf{B}=\left[\begin{array}{ccc}
10,7 & 0 & 0 \\
0 & 10,1 & 0 \\
0 & 0 & 20,3
\end{array}\right] \mathrm{kg}
$$

whereas the stiffness matrix, containing three unknown parameters, has the form

$$
\mathbf{K}=\left[\begin{array}{ccc}
k_{1}+k_{2} & -k_{2} & 0 \\
-k_{2} & k_{2}+k_{3} & -k_{3} \\
0 & -k_{3} & k_{3}
\end{array}\right] \frac{\mathrm{N}}{\mathrm{m}} .
$$

Thus, by virtue of formula (13), a heterogeneous system of three algebraic equations with three unknown parameters is obtained. The solution to this system is the sought-after parameters of the stiffness matrix $\mathbf{K}$, based on which the 
Table 2: Key results of experimental research.

\begin{tabular}{|c|c|c|c|c|c|c|c|}
\hline & & \multicolumn{6}{|c|}{ Measurement } \\
\hline & & No. 1. & No. 2 . & No. 3. & No. 4. & No. 5 . & No. 6 . \\
\hline \multirow{4}{*}{$\begin{array}{l}\text { Values of limit } \\
\text { expressions-formulas } \\
(11,12)\end{array}$} & $\begin{array}{l}Y_{1 \mathrm{c}} \\
(\mathrm{mm})\end{array}$ & 249.353 & 496.455 & 422.826 & 843.34 & 401.447 & 788.547 \\
\hline & $\begin{array}{l}Y_{2 c} \\
(\mathrm{~mm})\end{array}$ & 349.069 & 695.045 & 591.967 & $1,180.66$ & 562.036 & $1,103.95$ \\
\hline & $\begin{array}{l}Y_{3 c} \\
(\mathrm{~mm})\end{array}$ & 398.909 & 794.343 & 676.542 & $1,349.31$ & 642.335 & $1,261.64$ \\
\hline & $\begin{array}{l}\mathrm{Z}_{\mathrm{c}} \\
\left(\mathrm{mm} / \mathrm{s}^{2}\right)\end{array}$ & $6,283.19$ & $12,566.4$ & $10,000.0$ & $20,000.0$ & $10,000.0$ & $20,000.0$ \\
\hline \multirow{3}{*}{$\begin{array}{l}\text { Coefficients of the } \\
\text { stiffness matrix have been } \\
\text { determined based on } \\
\text { formula (13) }\end{array}$} & $\begin{array}{l}k_{1} \\
(\mathrm{~N} / \mathrm{m})\end{array}$ & $1,037.6$ & $1,042.31$ & 973.876 & 976.546 & $1,025.74$ & $1,044.4$ \\
\hline & $\begin{array}{l}k_{2} \\
(\mathrm{~N} / \mathrm{m})\end{array}$ & $1,918.93$ & $1,927.07$ & $1,800.51$ & $1,805.64$ & $1,896.4$ & $1,931.1$ \\
\hline & $\begin{array}{l}k_{3} \\
(\mathrm{~N} / \mathrm{m})\end{array}$ & $2,561.7$ & $2,571.53$ & $2,402.58$ & $2,409.76$ & $2,530.53$ & $2,577.21$ \\
\hline \multirow{3}{*}{$\begin{array}{l}\text { Natural frequencies of } \\
\text { the model have been } \\
\text { determined based on } \\
\text { formulas }(14,15)\end{array}$} & $\begin{array}{l}f_{1} \\
(\mathrm{~Hz})\end{array}$ & 0.665 & 0.667 & 0.644 & 0.645 & 0.661 & 0.667 \\
\hline & $\begin{array}{l}f_{2} \\
(\mathrm{~Hz})\end{array}$ & 2.343 & 2.349 & 2.270 & 2.274 & 2.330 & 2.351 \\
\hline & $\begin{array}{l}f_{3} \\
(\mathrm{~Hz})\end{array}$ & 3.929 & 3.937 & 3.806 & 3.811 & 3.906 & 3.942 \\
\hline
\end{tabular}

eigensystem is solved (14) and then the natural frequencies of the model are determined. Table 2 presents the most important results of experimental research from the point of view of the performed calculations. It is worth recalling that the natural frequencies of the model, identified by means of EMA, amount to $f_{1}=0.652 \mathrm{~Hz}, f_{2}=2.34 \mathrm{~Hz}, f_{3}=$ $3.92 \mathrm{~Hz}$.

One of the key problems related to the application of the method of identifying system parameters presented in this article was to obtain a dynamic response of the model in the form of displacements. This problem is related to the double integration of registered construction accelerations in order to obtain displacements. As we know, in such an operation, an unknown, parasitic trend appears, which can have a huge impact on the accuracy of further results. In addition, the recording of accelerations by the measurement instruments is performed discretely, with a certain sampling frequency, which also translates into the application of adequate data processing tools, for example, for the analysis of the registered signal in the frequency domain instead of the Fourier transform its equivalent discrete signals are used, that is, the discrete Fourier transform (DFT). The methods of discrete signal analysis and their processing and application of filters have been widely discussed, among others, in the works [13] and [14]. For the needs of experimental research carried out as a part of this work, an algorithm was developed in the Mathematica package for processing registered accelerations. In this algorithm, bandpass filters imposed on a signal transformed from the time domain into the frequency domain were applied. For this purpose, the DFT was used, discussed in detail in the work [13]. Then, the filtered signal was transformed back into the time domain in which the integration procedure was carried out with the simultaneous removal of integration constants. Finally, the plots of real displacements corresponding to the assumed generalized coordinates were obtained (see Fig. 1b). It should be mentioned, however, that these displacements are not described by continuous functions of time but constitute discrete sets of points, whereas each point contains a value corresponding to a given moment of time. Figures 5-10 in a sub-point "a" show displacement plots for all three stories of the model, which were obtained using the discussed algorithm, performing measurements from 1 to 6.

In subpoint " $\mathrm{b}$ " in Figures 5-10, the displacement plots obtained in accordance with formula (7) are presented, for which limit values were calculated in the further course of the procedure using formula (12). It is easy to notice (see Figs. 5b-10b) that the oscillations of the discussed displacements are shifted in relation to the time axis and occur around the constant values, which have been marked as $Y_{c}$ in formula (12). In addition, the character of each of these plots is analogous to free oscillations, and 

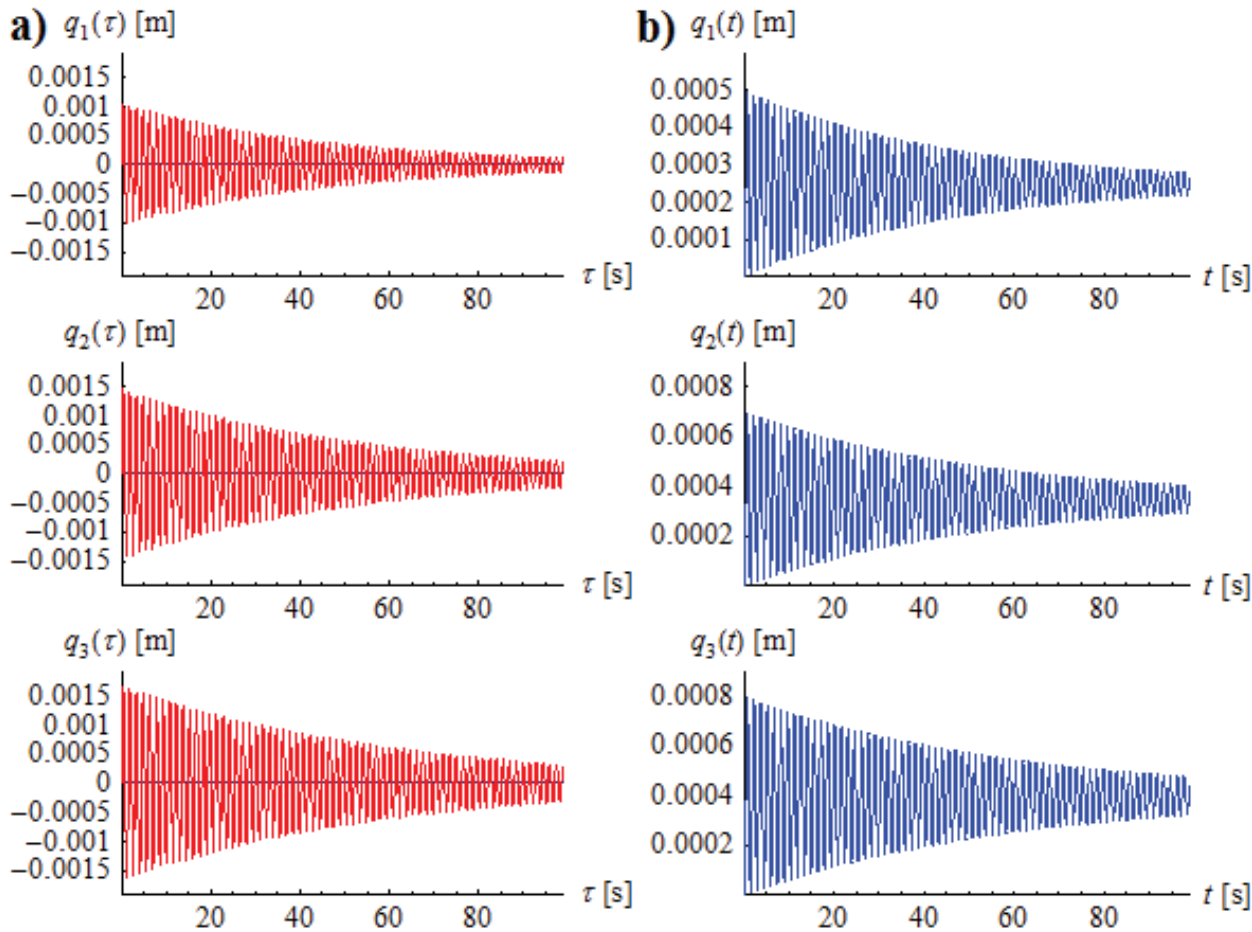

Figure 5: The results obtained from the first measurement: (a) displacement plots in the time domain $t$ and (b) displacements determined in accordance with formula (7).
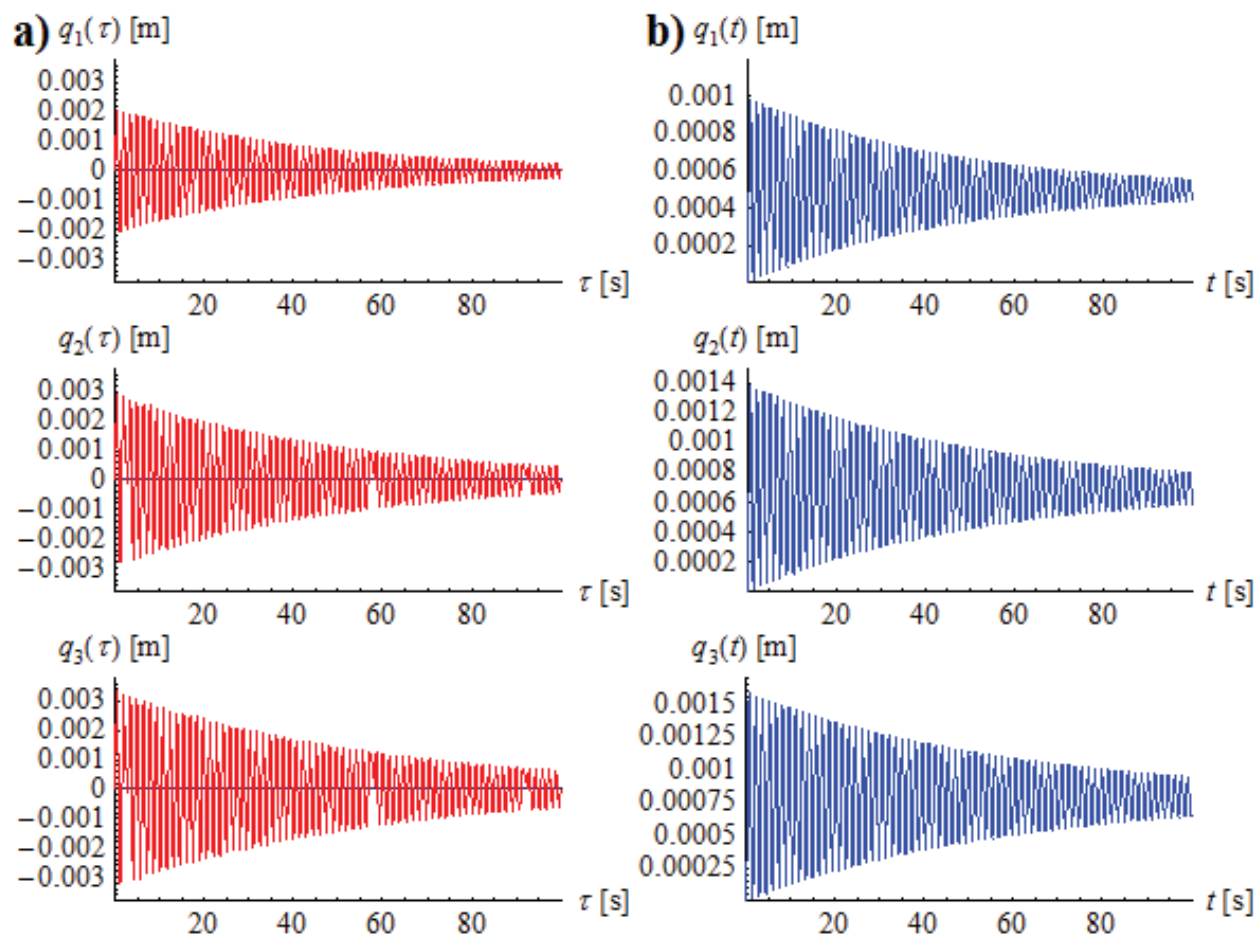

Figure 6: Results obtained from the second measurement: (a) displacement plots in the time domain $t$; and b) displacements determined in accordance with formula (7). 

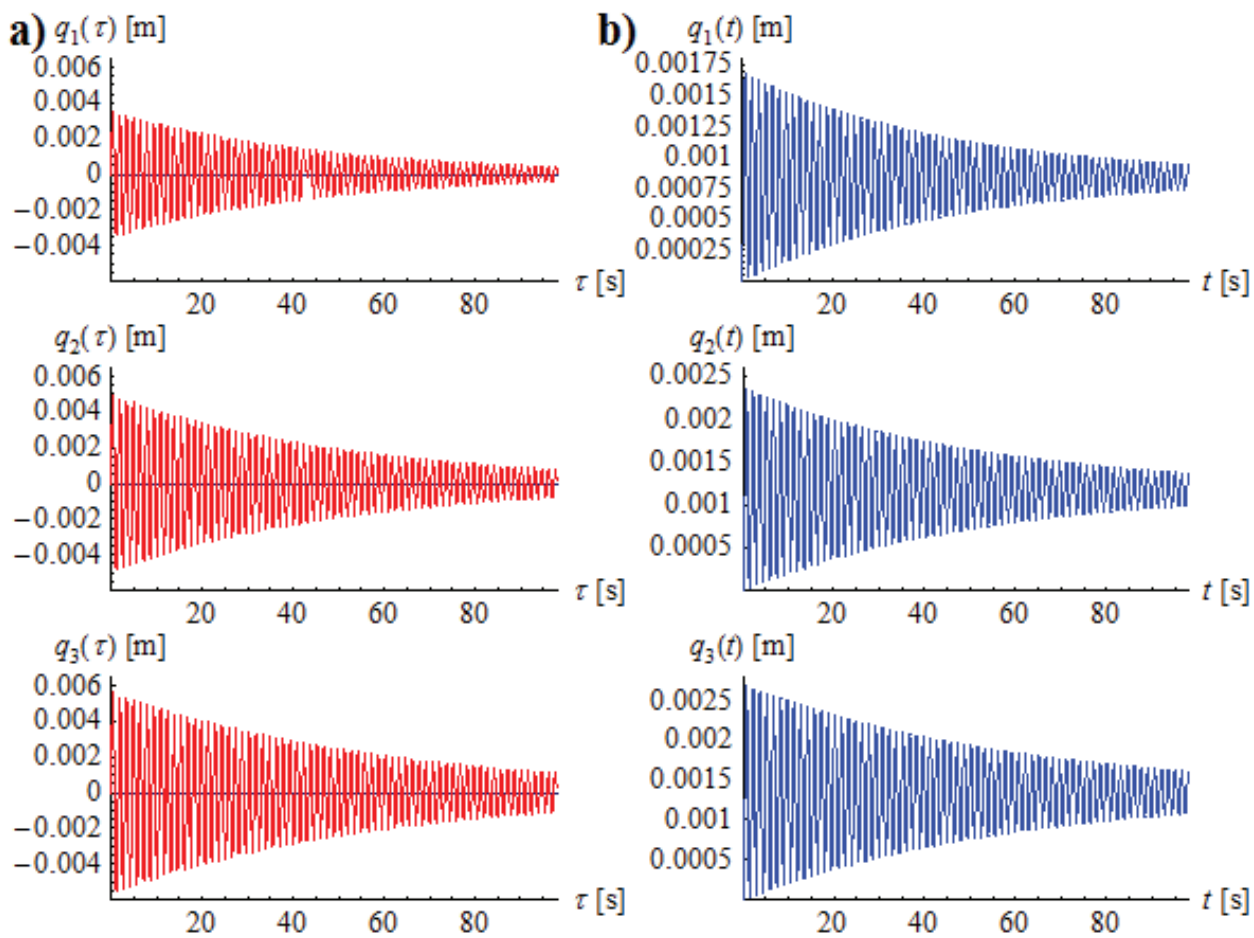

Figure 8: The results obtained from the fourth measurement: (a) displacement plots in the time domain $t$ and (b) displacements determined in accordance with formula (7).
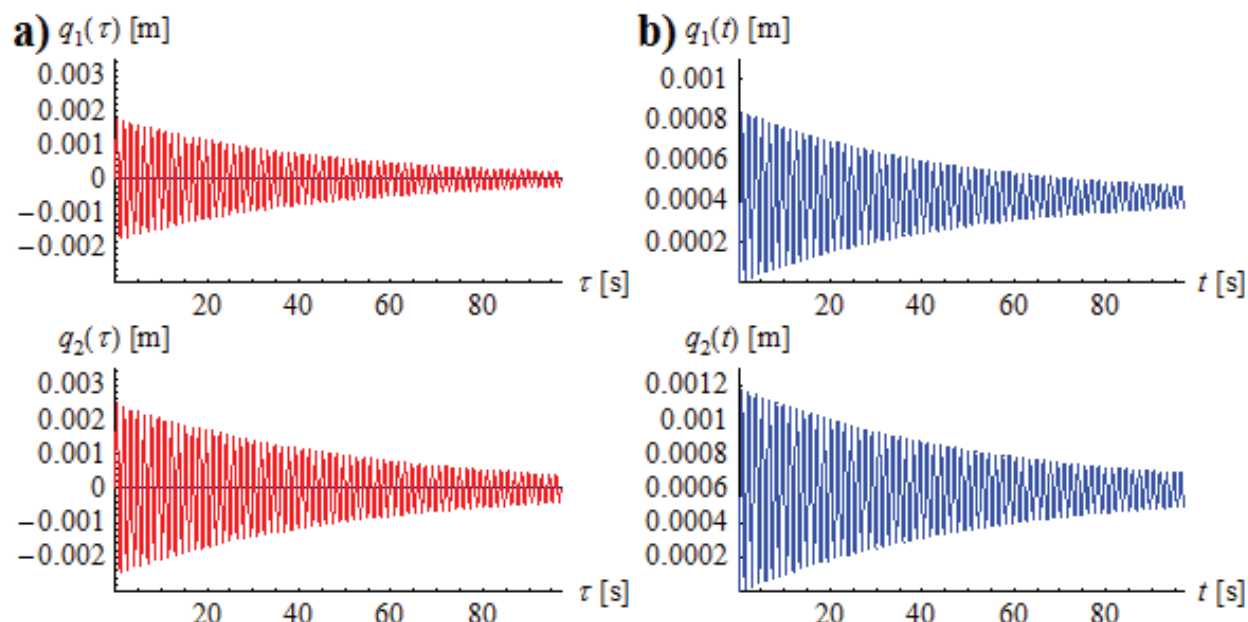

$$
q_{2}(t)[\mathrm{m}]
$$
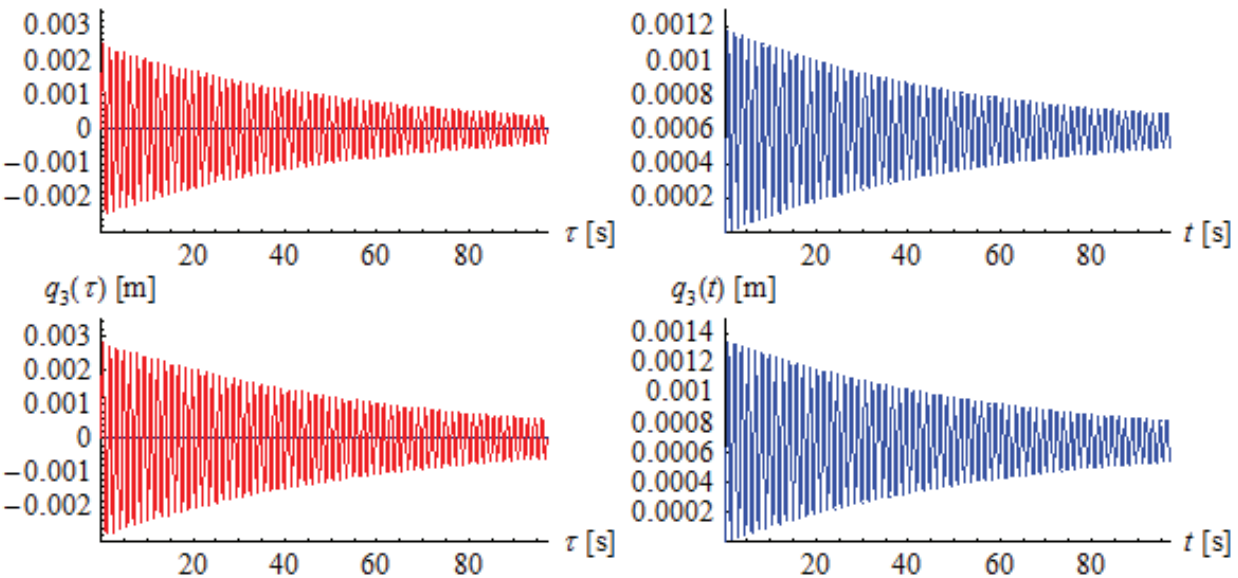

Figure 7: The results obtained from the third measurement: (a) displacement plots in the time domain $t$ and (b) displacements determined in accordance with formula (7). 

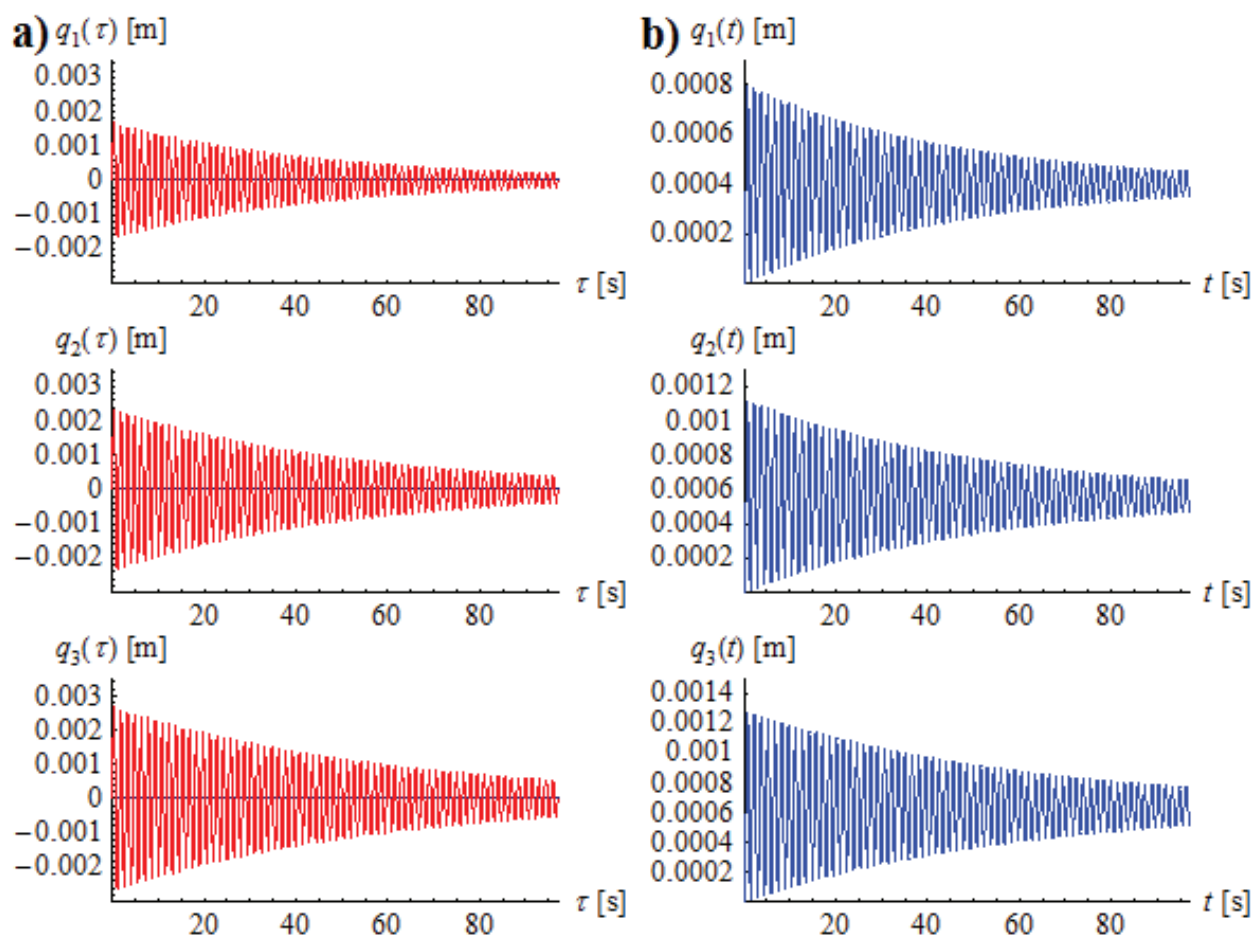

Figure 9: The results obtained from the fifth measurement: (a) displacement plots in the time domain $t$ and (b) displacements determined in accordance with formula (7).
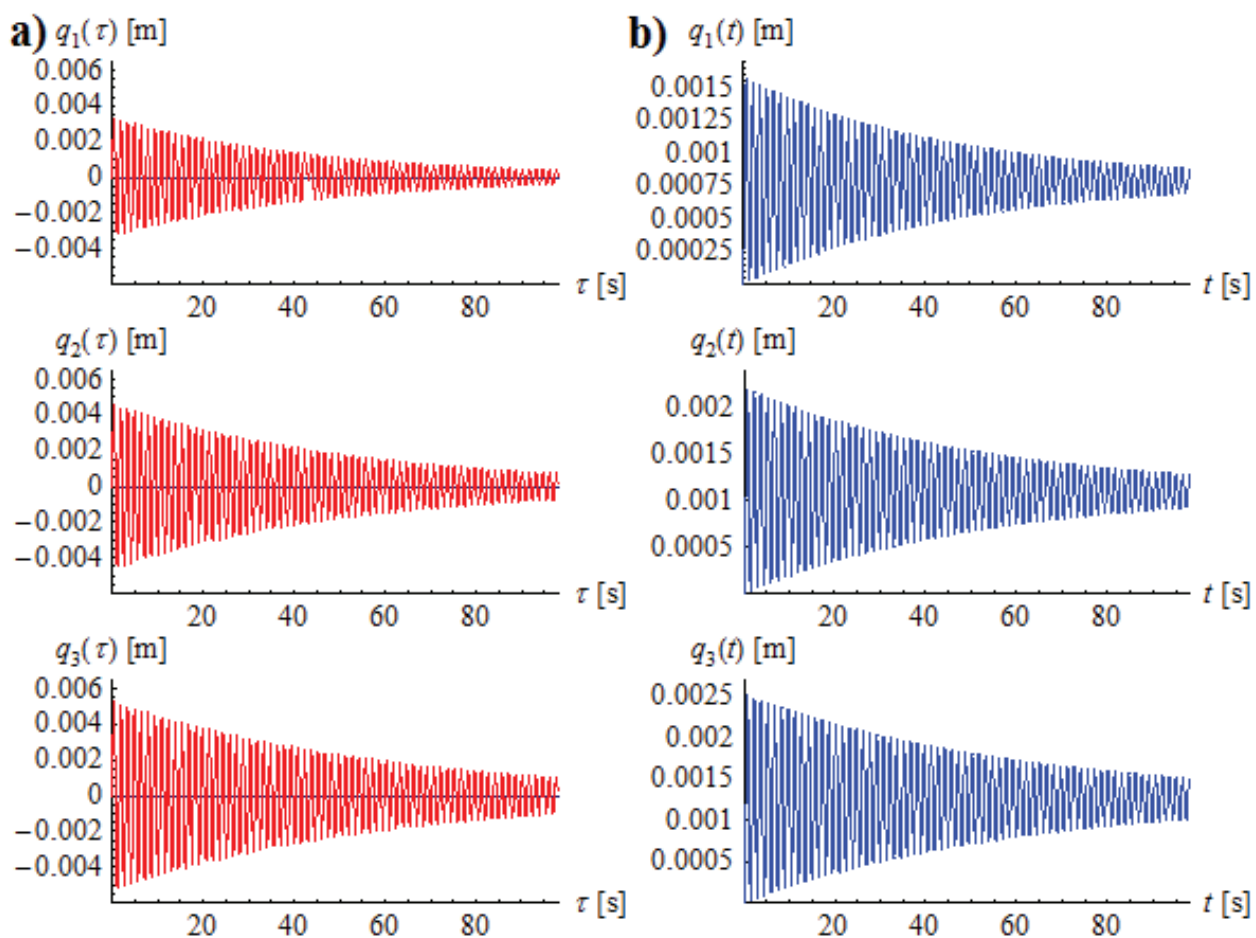

Figure 10: The results obtained from the sixth measurement: (a) displacement plots in the time domain $t$ and (b) displacements determined in accordance with formula (7). 
thus with time, the vibrations disappear and only the constant "static" value of the displacement remains. This fact may constitute experimental evidence, confirming the correctness of the theory formulated and proposed in the work [7] and of the methods for the identification of system parameters.

The presented results clearly show that the verified method provides results comparable with those obtained by the EMA in the form of the model's natural frequencies. When comparing the obtained frequency values to the reference values from the EMA, the relative error does not exceed $3 \%$, which may testify to the high effectiveness of the verified method. In addition, the conducted research has also shown that the verification of the modal parameters of the system by means of short-time kinematic excitation is possible. Despite revealing numerous similarities to the EMA, such approach may still constitute an alternative thereto. The basic argument supporting this thesis may be the fact that in the case of large objects (e.g., a tall building), it is impossible to excite vibrations through any kind of artificially induced impulses (e.g., with an impact hammer). On the other hand, short-time ground vibrations can be excited more easily, for example, by dropping a large mass tamper from a certain height in the vicinity of the tested object (cf. formation of gravel columns in the dynamic soil replacement method). In such cases, no external force is exerted on the tested object, while its vibrations are caused by a short-time kinematic input. As a result, some aspects of the application of the verified method are observable in practice. Once again, it is worth mentioning the work [6], in which the authors analyzed, among others, the method of identifying modal parameters of the system loaded with an earthquake.

\section{Summary and Conclusions}

This article presents the results of experimental research, the aim of which was to verify the theoretical considerations published in the work [7]. Six separate vibration measurements of the physical model were made, after which the input-output method was used to identify its modal parameters. In order to make the reference values of the model's natural frequencies available, the EMA and numerical analyses were performed beforehand on a calculation model defined based on the FEM. This work does not include the results of these studies together with the discussion, except for the values of natural frequencies, to which the natural frequencies obtained with the method proposed in the work [7] are compared. Owing to this, before the commencement of the research, the modal parameters of the physical model were known, which, in turn, largely facilitated the evaluation of the method proposed in the work [7].

Unfortunately, every experimental method has its major disadvantage and so does the one verified in this work, namely, it stipulates that structure displacement be available. With regard to real constructions, and therefore objects of considerable size, the measurement of displacements is extremely troublesome and sometimes even impossible. The difficulty results directly from the idea of displacement measurement, which consists in installing displacement sensors in the absolute reference system! It is obvious that for the vast majority of real construction objects, such measurements will not be possible. One may attempt to avoid the problem and measure deformations, subsequently obtaining displacements through geometrical relationships, but such approach may also be encumbered by excessive error. Thus the most common one remains, that is, the measurement of accelerations by means of accelerometers or, alternatively, the measurement of velocity, for example, by laser vibrometers. However, in the latter case, the vibrometer also needs an absolute reference system. The measurement of accelerations is the least troublesome for one simple reason-accelerometers are located in the neuralgic points of the structure; subsequently, the accelerations with which these points move are directly measured. As a result, no additional constructions to which accelerometers would have to be mounted are necessary. Unfortunately, with such an approach to the experiment, the issue of the double integration of accelerations immediately arises. This operation may appear to be seemingly trouble free, but in practice, it may cause many problems. First, registered accelerations are obtained in the form of a discrete set of points and not as a continuous function of time. Second, in order to obtain displacements, which will ultimately also constitute a discrete set of points, procedures analogous to those used in the calculation of the indefinite integral should be applied. This, in turn, causes the emergence of integration constants and, as a result, the appearance of the so-called parasitic trend. Third, because the measurements of accelerations and displacements were not conducted simultaneously, we will never achieve certainty that the displacements obtained by integration are the real ones that actually occurred in the structure.

The abovementioned advantages and disadvantages of the verified method, observed while conducting experimental research, allow for formulating the following conclusions: 
1. The theoretical considerations published in work [7], based on which the authors proposed the method for identifying modal parameters of the system subjected to short-time kinematic excitations, have been successfully translated and applied to experimental research conducted on a laboratory model. When applying this method, the obtained values of natural frequencies were very close to the reference values from the EMA.

2. By means of short-time kinematic excitations, the identification of the modal parameters of the system on a model scale is possible. This conclusion provides the grounds for surmising that the verified method could be applied to identifying the parameters of a real object, provided it would be possible to induce short-term ground vibrations in a way that would not cause any damage to the tested structure.

3. The disadvantage of the verified method is the requirement to know the displacements of the structure caused by the movement of its mainstay part. The direct measurement of displacements on the scale of a real construction object may be very difficult or even impossible to perform. In turn, the measurement of accelerations implies difficulties related to the calculation of indefinite integrals.

4. In case of applying the verified method to the identification of the modal parameters of a real construction object, one of the key theoretical assumptions about "zero initial conditions" may constitute a serious problem, and, therefore, another disadvantage. Obviously, such an assumption may not be possible to fulfill because, in the natural environment, construction objects are constantly exposed to vibration excitement. Wind gusts, vehicle traffic adjacent to the building, machine operation, and so on may prevent the tested object from being motionless.

5. The experimentally verified method of identifying dynamic system parameters can be used in practice. However, this requires proper planning of the experiment so as to minimize the disadvantages of the method. If, with respect to the real structure, tests can be carried out, such as during laboratory experiments, the verified method can be an alternative to other experimental methods in the field of identification of modal parameters.

\section{References}

[1] Alvin, K. F., Robertson, A. N., Reich, G.W. \& Park, K.C. 2003. Structural system identification: from reality to models, Computers and Structures, 81,1149-1176.

[2] Cunha, A., Caetano, E. 2006. Experimental Modal Analysis of Civil Engineering Structures, Sound and Vibration, (2006, June), pp. 12-20.

[3] Grosel, J., Sawicki, W., Pakos, W. 2014. Application of Classical and Operational Modal Analysis for Examination of Engineering Structures, Procedia Engineering, 91, pp. 136 - 141.

[4] Grosel, J., Sawicki, W., Wojcicki, Z. 2009. The identifications of modal parameters of a large industrial structure, Proceedings of The 3rd International Operational Modal Analysis Conference (IOMAC), Ancona, Italy.

[5] Harris, C. M., Piersol, A. G. 2002. Shock and Vibration Handbook. $5^{\text {th }}$ ed. USA: The McGraw-Hill Companies Inc.

[6] Imai, H., Yun, C. B., Maruyama, O. \& Shinozuka, M. 1989. Fundamentals of system identification in structural dynamics, Probabilistic Engineering Mechanics, 4, 162-173.

[7] Jarczewska, K., Koszela, P., Śniady, P. \& Korzec, A. 2011. Identification of the structure parameters using short-time non-stationary stochastic excitation, Journal of Sound and Vibration, 330, pp. 3352-3367.

[8] Ljung, L. 1987. System Identification. Theory for the User, Prentice-Hall PTR.

[9] Lus, H., Betti, R., Longman, R.W. 1999. Identification of linear structural systems using earthquake-induced vibration data, Earthquake Engineering and Structural Dynamics, 28, pp.1449-1467.

[10] Majcher, K. 2014. The Influence of Changing Stiffness Characteristic of a Semi-Active Tuned Mass Damper onto the Effectiveness of Vibration Reduction in a Plate-Column Structure, Procedia Engineering, 91, pp. 124 - 129.

[11] Pappalardo, C. M., Guida, D. 2018. System Identification and Experimental Modal Analysis of a Frame Structure, Engineering Letters, Vol. 26, No. 1, pp. 56-68.

[12] Pridham, B.A., Wilson, J.C. 2004. Identification of base-excited structures using output-only parameter estimation, Earthquake Engineering and Structural Dynamics, 33, pp. 133-155.

[13] Shenoi, B. A. 2006. Introduction to digital signal processing and filter design. Published by John Wiley \& Sons, Inc., Hoboken, New Jersey.

[14] White, S. 2000. Digital Signal Processing: A Filtering Approach, $1^{\text {st }}$ ed., Delmar Cengage Learning, ISBN:0766815315.

[15] Wójcicki, Z., Grosel, J., Sawicki, W. 2014. Experimental dynamic investigation of structures, DWe, Wrocław, [in Polish]. 